\title{
Zur medizinischen Versorgung der Berner Landschaft im 18. Jahrhundert*
}

Von Marta Meyer-Salzmann

\section{Hausmedizin}

Als erste Hilfe wurde dem Kranken im 18. Jahrhundert Hausmedizin zuteil. Familienmitglieder griffen in dieser Absicht ein, und erst, wenn die Heilung in absehbarer Zeit nicht gelang, suchte man einen Heilpraktiker auf. Doch bei einem schweren Unfall war die sofortige Hilfe eines Wundarztes sehr geschätzt. Formschöne Hausapotheken aus Herrschaftshäusern, wie sie im Schweizerischen Pharmazie-Historischen Museum in Basel zu sehen sind, bezeugen die Wichtigkeit der Hausmedizin.

Wir beziehen uns hier auf zwei handschriftlich angelegte Rezeptsammlungen aus dem Emmental ${ }^{1}$. Da gedruckte Bücher mit medizinischen Anleitungen teuer waren, suchte man durch Abschriften in den Besitz dienlicher Rezepte zu gelangen.

Viele Heilmittel bestanden aus Kräutern, Beeren, Samen, Rinden, Wurzeln und Gewürzen. Daraus konnten am häuslichen Herd Tees, Bäder, Breie und Salben bereitet werden. Gewisse Salben enthielten Bleiglätte, Goldglätte, Cuprum sulfuricum oder Zincum sulfuricum. Tierbestandteile, vor allem Fette, und auch Exkremente fehlten nicht. Man hat die Verwendung von Exkrementen später der Volksmedizin übelgenommen. Doch bildete ihre Anwendung eine Ausnahme. Auch der studierte Mediziner gebrauchte hie und da noch Urin. Daß Ausscheidungen gesunder Lebewesen dem Kranken Fehlendes ersetzen könnten, wollte man auch im 18. Jahrhundert nicht ganz von der Hand weisen ${ }^{2}$.

Es sind in diesen Rezeptsammlungen Maßnahmen vorgesehen bei Wunden und Furunkeln, Verrenkungen und Leistenbrüchen, bei Schmerzen an verschiedenen Stellen, bei Übelkeit und Brechreiz und vielen weiteren Beschwerden. Es fanden auch die Mondphasen Beachtung, vor allem für das Kräuterpflücken. Durch die Rezepte schimmert noch die Heilkunst der alten Ägypter, Babylonier, Griechen, Germanen und die der Klöster.

${ }^{*}$ Herrn Prof. Dr. med. Heinrich Buess ehrerbietig zugeeignet.

Gesnerus 1/2 (1981) 


\section{Apotheken}

An der häuslichen Heilkunst hatte der Apotheker Anteil. Zutaten zu Heilmitteln konnten bei ihm bezogen werden; man brachte ein Rezept zur Anfertigung oder kaufte fertige Arzneien. Gegen Ende des 17. Jahrhunderts besaß Burgdorf zwei Apotheken ${ }^{3}$. Im 18. Jahrhundert diente Michel Schüppachs gut ausgestattete Apotheke in Langnau einem weiten Kreis ${ }^{3,5}$.

In Burgdorf war die erste Apotheke aus einer Gürtlerwerkstatt hervorgegangen. Johann Ulrich Grimm hatte metallene Gürtelschnallen verfertigt. Seit dem Mittelalter waren Metallwerkstätten oft zugleich Experimentierorte der Alchimie. Die chemische Behandlung von Metallen ergab die Beziehung zur Heilmittelchemie.

Im 18. Jahrhundert ordnete man die Arzneien in Gruppen. Aufsaugende Mittel sollten innerlich im Körper oder äußerlich bei Wunden Säuren aufsaugen. Ferner unterschied man zusammenziehende und eröffnende oder säfteausleerende Mittel, also Laxier- und Brechmittel. Weitere Einteilungen waren: Säfte verändernde Mittel, die zum Beispiel dickes Blut verdünnten, schmerzstillende und erquickende Mittel. Die Arzneien wurden auf Körperteile oder Organe oder einfach auf Symptome bezogen ${ }^{4}$. In den Apotheken wurde destilliert, sublimiert, kristallisiert, gepreßt, extrahiert, gekocht, geschmolzen, gelöst und gemischt. Man erstrebte verfeinerte Zubereitungen, um die Drogen bekömmlicher zu machen. Der Dießbachbalsam, dessen Rezept von ausländischen Hofapothekern stammt, wurde eine emmentalische Spezialität und ist heute noch erhältlich. Er besteht aus ätherischen Ölen, Wacholderbeeren, Perubalsam und Chinarinde ${ }^{3}$.

Der zukünftige Apotheker mußte sich über Lateinkenntnisse ausweisen, bevor er eine Lehre in einer Apotheke antreten konnte ${ }^{6}$. In Burgdorf hatte der eine Apotheker sogar in Leiden Medizin studiert und war mit dem Doktortitel heimgekehrt. Der andere, Apotheker und Arzt genannt, machte seine Studien in Straßburg ${ }^{7}$. Als Existenzgrundlage diente ihnen die Apotheke mit der Kundenberatung.

\section{Geburtshilfe}

Von großer praktischer Bedeutung waren die Hebammen. Die Witwe des Dr. med. David Grimm von Burgdorf - er war Leibarzt des Abtes in Muri wurde in Burgdorf Stadthebamme. Einem Bericht zufolge hatte sie große 
Erfahrung und verrichtete viele Kurbehandlungen ${ }^{8}$. - In Oberdießbach wirkte Frau Margaritha Fischer-Klossner als eine «von einer Wohl Edelgebornen Herrschafft geordnete Hebamme». Sie half vielen Armen ohne Entgelt und war mit allerhand gebrannten Wassern, Wermutextrakt und dergleichen versehen, womit sie denen, die es gesundheitshalber nötig hatten, behilflich war ${ }^{9}$. - Die beiden Hebammen suchten sich also auch in der inneren Medizin nützlich zu erweisen.

Die Behörden wählten und überwachten die Hebammen. In der klassischen Antike gab es praktische und theoretische geburtshilflich-gynäkologische Schriften, und seit dem 16. Jahrhundert waren hierzulande allgemein «nützliche Büchlein» verbreitet, die den Hebammen zum fleißigen Studium empfohlen wurden ${ }^{10}$. In einem Bauernhaus in Waldhaus bei Lützelflüh wurde um 1920 ein Buch aus dem Jahr 1725 versteigert, das den Titel hat: «Neu-entdeckte Heimligkeiten Des Frauenzimmers». Darin stehen Angaben über den Bau der Frau, über die Frauenkrankheiten und über die Geburt. Es wird geraten, daß man bei schweren Geburten, so es möglich sei, einen geübten Medicus oder Chirurgus rufen solle ${ }^{11}$. Doch gute Hebammen verstanden bei falschen Lagen des Kindes im Mutterleib Wendungen auszuführen und kannten eine Reihe von Maßnahmen, um schwierige Geburten zu erleichtern ${ }^{12}$.

Um 1650 wird ein Meister der Schnitt- und Wundarzneikunst, Samuel Neukommet in Signau, als guter Geburtshelfer genannt ${ }^{13} .1759$ wurde ein Kaiserschnitt in Langnau ausgeführt. Die Gebärende war noch sehr jung, fast neunjährig. Der Name des Chirurgen wird nicht erwähnt ${ }^{14}$. Möglicherweise war es Michel Schüppach. (Auf dessen Wirken wird hier nicht näher eingegangen, da eine ausführliche Biographie vorliegt ${ }^{5}$.) In Sumiswald besorgte sieben Jahre später der «Möösli-Schärer» einen solchen Schnitt. Er wurde dafür gut bezahlt ${ }^{15}$.

In Straßburg war 1737 die Gebäranstalt zum Institut für Studierende der Medizin geworden ${ }^{16}$. Manche Emmentaler Wundärzte ließen sich in Straßburg weiterbilden, und man darf annehmen, daß sie sich bei schwierigen Geburten immer besser bewährten. In Bern kam es 1781 zur ersten Hebammenschule mit dreimonatigen Kursen ${ }^{17}$. 


\section{Der hochschulgebildete Arzt}

Seit dem Mittelalter gab es zwei ärztliche Stände, einerseits die an Hochschulen ausgebildeten Ärzte, andererseits die handwerklich geschulten Praktiker. Die aus Hochschulen hervorgegangenen waren zuweilen vielseitige Gelehrte. Bekannt sind Johann Jakob Scheuchzer in Zürich und Albrecht von Haller in Bern ${ }^{18}$. Akademiker forschten am Leichnam, führten aber, von Ausnahmen abgesehen, keine Operationen an Lebenden aus. Der studierte Arzt betätigte sich praktisch in der inneren Medizin ${ }^{19}$. Ein sicheres Einkommen besaß er oft durch ein Amt im Staatswesen oder durch einen Lehrauftrag an einer Hochschule, wobei er Studierende in sein Haus aufzunehmen pflegte ${ }^{20}$. Begehrt war die Stelle eines Stadtarztes. In Bern überwachte er die Apotheken, Gefängnisse, Spitalhäuser und die von der Stadt angestellten Wundärzte und Chirurgen; auch mußte er Maßnahmen bei Seuchen ergreifen - und Seuchen gab es viele. Im 18. Jahrhundert hatte es in Bern vier Physikate, und sie waren mit sechs Ärzten besetzt ${ }^{21}$.

Der erste studierte Arzt Burgdorfs, Dr. med.Johannes Kupferschmid ${ }^{22}$, geboren 1691, gehörte zu den Ausnahmen, denn er übte nicht allein innere Medizin, sondern auch Chirurgie aus. In begeisterten Sätzen hatte er sich schon in seiner Basler Dissertation für die Chirurgie eingesetzt und sich mit scharfen, spöttischen Worten gegen jene Ärzte gewandt, die sich scheuten, ihre wohlgepflegten Hände bei der Wundbehandlung zu beschmutzen. Der 24jährige Doktorand konnte die Worte wagen, denn er wußte sich von seinem berühmten Lehrer Theodor Zwinger (1658-1724) unterstützt. Die ersten wundärztlichen und chirurgischen Erfahrungen hatte er während des zweiten Villmerger Krieges 1712 im Lenzburger Lazarett gesammelt. Johannes Mathys (1676-1749), ein Burgdorfer Chirurg, führte dort ein erfolgreiches Behandlungsverfahren ein, indem er in warmem Weingeist getränkten Verbandstoff auf die Wunde legte oder den Weingeist mit einem Röhrchen direkt in die Wunde goß. Kupferschmid gibt dazu die Erklärung, dass Alkohol und fester Wundverband die Säftebewegung aufheben und zersetzende Luft abwehren.

Nicht nur Paris, Berlin und Wien kannten den Skandal wegen Leichenraubes, sondern auch Burgdorf. Sektionen waren an den schweizerischen Lehranstalten immer noch spärlich erlaubt. Johannes Kupferschmid, der Chirurg Mathys und der Medizinstudent Andreas Grimm, Sohn des Apothekers, sezierten 1729 heimlich nachts im Gartenhaus bei Kupferschmid einen tödlich Verunfallten. Der entleerte Sarg wurde mit Wurzelstöcken und 
Steinen gefüllt. Das Geschehnis kam an den Tag; auf der Kanzel wetterte Pfarrer Johann Rudolf Gruner gegen ein solches Verbrechen am christlichen Menschen. Ein Gutachten des bernischen Rates entschied, daß Kupferschmid und Grimm sich durch eine Schrift verteidigen und die Wichtigkeit des Studiums am menschlichen Körper darlegen durften. Trotzdem erfolgte 1730 die Verurteilung zu hohen Bußen. Der Diebstahl mußte gesühnt werden. Kupferschmids weiteres Leben stand unter keinem glücklichen Stern. Er verbrachte seine letzten Jahre unter dürftigen Umständen in Paris und starb dort 1750 .

Das anatomische Übungsfeld des Handwerksarztes, vor allem des Landwundarztes, war der Tierkadaver. Für die Abklärung von Kriminalfällen wurde der Landwundarzt auch zur Sektion des menschlichen Körpers herangezogen ${ }^{23}$.

\section{Badstuben}

Öffentliche Badstuben entwickelten sich seit der zweiten Hälfte des Mittelalters so sehr, daß der Beruf des Baders und des Wundarztes sich mischten. Unter demselben Dach wurden im 16. Jahrhundert Schwitz- und Kräuterbäder, Wundbehandlungen, das Zähneziehen, Aderlassen und Schröpfen und die Barbierpflege besorgt ${ }^{24}$.

Im Emmental gab es noch durch das ganze 18. Jahrhundert Badstuben, worin vor allem Heilbäder und Schröpfen zur Anwendung gelangten. Manchmal waren sie mit einem Mineralbad verbunden. Hie und da wurde die Badstube von einem Wundarzt geführt; aber sie stellte kein medizinisches Zentrum mehr dar ${ }^{25}$.

\section{Die Chirurgische Societät und die Handwerksärzte}

Gegen Ende des 15. Jahrhunderts hatten sich in der Stadt Bern die Wundärzte zu einer Meisterschaft vereinigt, die sich Chirurgische Societät nannte ${ }^{26}$. Sie anerkannte 1663 die Gesellenordnung des Heiligen Römischen Reichs Deutscher Nation als verbindlich ${ }^{27}$. Dies war wesentlich für den Bildungsaustausch junger Leute über die Landesgrenzen hinaus. Vorgeschrieben wurden drei Jahre Lehrzeit und drei Jahre Wanderschaft, später noch ein zusätzliches Jahr für Chirurgie. Dann konnte man vor der Chirurgi- 
schen Societät die Meisterprüfung ablegen. Die Societät waltete auch als Standesgericht, behandelte Klagen über Kunstfehler, Arzthonorare oder unbotmäßige Gesellen. Ihr stand vom bernischen Rat das Recht zu, Kurpfuscher zu büßen, eine Ermächtigung, von der sie immer wieder Gebrauch machte ${ }^{28}$. Die Societät hatte sich 1627 an den bernischen Rat gewandt, die Handwerksmeister müßten mit Bedauern zusehen, daß ohne Unterschied zu Stadt und Land, sowohl an Weibs- wie Mannspersonen, mit ungewaschenen Händen die edle Kunst der Arznei ausgeübt und der Mensch an seinem Leib ganz übel traktiert werde. Ihr Anliegen sei, eine Satzung zu beraten, um das Stümpelvolk abzuschaffen und erfahrene Meister heranzubilden ${ }^{29}$. Zur Wundarzneikunst gehörte die Behandlung von Verletzungen, Knochenbrüchen und Luxationen, zur Chirurgie der Bruch- und Steinschnitt, der Starstich, die Hasenschartenoperation und die Amputation.

Die 1628 neu gefaßte Ordnung der Chirurgischen Societät nennt die Einverleibung von Meisterschaften auf dem Land, auch von Burgdorf ${ }^{30}$. 1707 kam Langenthal mit Huttwil hinzu ${ }^{31}$.

Die Meisterprüfung mußte der Kandidat im 18. Jahrhundert vor neun Examinatoren ablegen. Darunter befanden sich der älteste Stadtarzt, InselWundärzte und -Chirurgen und weitere Meister der Schnitt- und Wundarzneikunst sowie der Seckelmeister ${ }^{32}$. Im Fähigkeitsausweis stand oft die Ermahnung, daß der passierte Meister sich weiterhin guter Autoren bedienen solle. Es gab von Wundärzten geschriebene Chirurgie- und Wundarzneibücher, ferner populärwissenschaftliche sogenannte Kunstbücher und deutsch verfaßte oder ins Deutsche übersetzte Originalwerke von Gelehrten ${ }^{33}$. Ein limitierter Meisterbrief verbot die Ausübung der Chirurgie; ein unlimitierter berechtigte dazu. In der zweiten Hälfte des 18. Jahrhunderts schloß sich noch eine Prüfung in Geburtshilfe an. Da die Vorschriften der Societät nicht immer eingehalten wurden, kam es oft zu Klagen.

An der Universität Straßburg betraf die Prüfung des Chirurgen 1757 das Skelett, Frakturen, Luxationen, Bandagen und weitere Fragen von Chirurgen und Medizinern; hinzu kamen drei oder vier Operationen am Kadaver mit Erläuterungen, was vor, während und nach der Operation geschehen solle ${ }^{34}$.

Auch in Bern wurde Chirurgie praktisch geprüft. Michel Schüppach war in der Chirurgia practica gut bewandert; in der Chirurgia theoretica sollte er sich noch festigen ${ }^{35}$. Nicht jeder bestand das Examen; wenige wurden für den ärztlichen Beruf gänzlich abgelehnt; andere sollten sich später für dieses 
oder jenes Fach wieder stellen. Im Juli 1762 erhielt ein Kandidat die Aufforderung, daß er sich wenigstens bis künftigen Frühling in Straßburg zu chirurgischen Kollegien aufhalten solle ${ }^{36}$. Die Prüfung kostete den Kanditaten 150 bis 180 Fr., nach Geldwert um 1960 umgerechnet. Oft spendete der neue Meister den Examinatoren noch ein Nachtessen ${ }^{37}$. Der empfangene Meisterbrief erlaubte dem Handwerksarzt, zu praktizieren und Lehrlinge auszubilden.

Wir nennen hier drei Emmentaler aus der gebildeten Schicht, die in den Manualen der Chirurgischen Societät eingetragen sind: 1685 hatte der Sohn des Prädikanten zu Eriswil, Hans Heinrich Gysi, der bernischen Societät gemeldet, daß er die Kunst der Wund- und Schnittarznei, die Ophthalmia und Lithotomia erlernt habe und sich examinieren lassen möchte ${ }^{38} .1722$ wurde der Sohn Wolfgang des Dr.med. Wolfgang Christen, der von 1710-1745 Stadtarzt in Bern war und aus dem Pfarrhaus in Krauchthal stammte, als Lehrling angenommen ${ }^{39}$. Samuel Kupferschmid, Sohn des uns bekannten Dr. med. Johannes Kupferschmid in Burgdorf, ersuchte 1746 um Aufnahme in die Meisterschaft ${ }^{40}$.

Von Michel Schüppach ausgebildete junge Leute haben die Meisterprüfung bestanden und bildeten dann selber wieder Lehrlinge aus ${ }^{41}$. Bei Schüppachs Schwiegersohn, Friedrich Brom, der in Langnau praktizierte, beendigte Christian Obmann von Wachseldorn 1767 eine Wundarztlehre und ging 1769 zur Weiterbildung nach Straßburg. Er ließ sich in Wachseldorn nieder, in jener einsamen Landschaft, in der einst Michel Schüppach seine Lehre gemacht hatte. Aus den Manualen nennen wir noch Ulrich Locher, Wundarzt in Burgdorf. Er kurierte einen am 5. März 1798 auf dem Fraubrunnenfeld am Ober- und Unterschenkel schwer verletzten Füsilier. Locher war 1783 in Straßburg gewesen, gemeinsam mit Alexander Burger von Sumiswald ${ }^{42}$. Nach den Matrikeln der Universität Straßburg gingen in der zweiten Hälfte des 18. Jahrhunderts aus dem Emmental dorthin zur Ausbildung: vier von Großhöchstetten, je zwei von Burgdorf, Langnau und Huttwil, je einer von Steffisburg (aus Biglen gebürtig), von Oberdießbach, Walkringen, Oberburg, Hasle, Heimiswil, Lützelflüh, Sumiswald, Signau und Röthenbach. Die Liste ist möglicherweise nicht vollständig, da in den Matrikeln nicht alle Studierenden erfaßt wurden, teils wegen ungenauer Ortsangaben, teils weil jene, die noch als Gesellen arbeiteten, nicht eingetragen $\operatorname{sind}^{53}$.

Die Handwerksärzte wurden mit ehrenvollen öffentlichen Ämtern betraut. In Burgdorf waren im 18. Jahrhundert vier von ihnen Burgermeister, 
andere Ratsmitglieder. Auf dem Land wirkten manche als Armenvögte, Chorherren, Gerichtssäßen und Weibel ${ }^{43}$.

Die Barbierarbeiten waren oft ein Stein des Anstoßes. Vor allem die städtischen Wundärzte wehrten sich für diese Einkommensquelle gegen die Bader. Die Landwundärzte hatten mehr Erwerbsmöglichkeiten; sie besaßen meistens Land, nahmen oft Patienten zur Pflege in ihr Haus auf, führten also eine Art Privatklinik, und durften in innerer Medizin praktizieren. Beim Militär dagegen lehnten sich Wundärzte gegen die Verfügung des Rasierens auf. In einem Traktat von 1758 über Dienstverhältnisse im Regiment steht, man solle die Feldscherer des Rasierens entheben ${ }^{44}$. Ein tüchtiger Wundarzt, Johann Ludwig Hommel ${ }^{45}$, Pfarrerssohn aus Deutschland, hatte als Geselle in Straßburg die Ausbildungsmöglichkeiten der dortigen Universität genutzt, dann als Geselle des Inselchirurgen Niclaus Wernier in Bern von 1732 bis 1736 die Behandlung Verletzter übernommen und anatomische Arbeiten ausgeführt. Er war glücklich, als er vom «verdrießlichen und zeitverderblichen Bartscheren» befreit wurde. Hommel wurde Prosektor und Dozent in Straßburg, Lehrer von Akademikern und Wundärzten. Mit Albrecht Haller stand er in freundlicher Beziehung.

Die von den Meistern ausgestellten Lehrbriefe heben weder den guten Haarschnitt noch die Sorgfalt beim Rasieren hervor, sondern bilden ärztliche Ausweise ${ }^{46}$. Die Verschönerungskunst nahm der Perruquier für sich in Anspruch. Der seit dem Mittelalter gebräuchliche Name Schärer für Wundarzt und Chirurg kommt zwar vom Haarescheren, ist aber irreführend. Die Kurpfuscher in der ersten Hälfte des 19. Jahrhunderts, wie wir sie aus Gotthelfs «Anne Bäbi Jowäger» kennen, sind nicht mit den Handwerksärzten des 18. Jahrhunderts identisch ${ }^{47}$.

Im 18. Jahrhundert erscheint als etwas unklares Gebiet die Ausübung der inneren Medizin. Der gelehrte Arzt war dazu berechtigt; der Apotheker fühlte sich dazu berufen; aber auf dem Land, wo kein Akademiker praktizierte, war der Wundarzt für diesen Bereich zuständig. Der junge Mann lernte innere Medizin von seinem Meister und durch Bücher, die mitunter von Apothekern verfaßt worden waren. Auch konnte er aus Pharmakopöen pharmazeutische Kenntnisse erwerben.

Die Diagnose stellten studierte Ärzte und Handwerksärzte nach den beobachteten Symptomen und nach dem, was der Patient oder ein ihm Nahestehender erzählte. Die Harnschau war inbegriffen, obwohl sie nur beschränkte Fingerzeige gab, da chemische Untersuchungen noch wenig entwickelt waren ${ }^{48}$. 
In Langenthal brachte die Familie Mumenthaler Handwerksärzte mit erweiterter Ausbildung hervor. Der angehende Wundarzt Johann Georg Mumenthaler immatrikulierte sich 1753 an der Universität Straßburg und blieb dort fünf Monate ${ }^{49}$. Er schrieb die Vorlesungen auf, vermutlich nach Notizen, und es entstand ein handschriftliches Buch von 354 Seiten über innere Medizin; es ist noch erhalten. Die Krankheiten sind in 67 Gruppen eingeteilt. Jeder Gruppe gehen zeitgemäße Erklärungen über Symptome, Ursache und Wirkung voraus; es folgen für die einzelnen Krankheiten die Behandlungsvorschriften und die Rezeptierung von Medikamenten. Erwähnt sind Hippokrates (geboren 460 vor Christus), Thomas Sydenham (1624-1689), der ein überzeugter Hippokratiker war, und Herman Boerhaave (1668-1738), der Kliniker in Leiden. Es kommt griechische Säftelehre von den herrschenden Körpersäften und ihren Abwandlungen zum Ausdruck. Jede Behandlung in diesem Buch verlangt ohne Ausnahme erstens die Reinigung des inneren Körpers von krankmachenden Säften, Säuren oder Schärfen durch Laxier- und Brechmittel, durch Klistiere, durch Adlerlaß und schweißtreibende Mittel. Bei manchen Krankheiten wurden sie der Reihe nach und zu wiederholten Malen allesamt gebraucht. Bäder und Diät spielen in der Krankenbehandlung eine große Rolle. Heilende, lindernde und tonische Mittel, zum Teil mit fremdländischen Drogen, wie sie die Pharmakopöen erwähnen, sind aufgeführt.

Die Mumenthaler hinterließen eine gut ausgestattete Bibliothek, die die Ausübung der Handwerksmedizin veranschaulicht ${ }^{50}$. Unter den Mumenthalern waren zwei als Wundärzte und Apotheker tätig; einer veröffentlichte zwei kleinere medizinische Bücher; ein anderer studierte ebenfalls in Straßburg und auch in Berlin; dieser wurde Militärarzt im Schweizerregiment von Diesbach und Schiffsarzt auf einem holländischen Kriegsschiff. Vier Ärzte Mumenthaler ließen sich von der Chirurgischen Societät prüfen, erhielten den Meisterbrief und bildeten selber Lehrlinge aus ${ }^{51}$.

\section{Die Ausbildung an der Universität Straßburg}

An der Universität Straßburg ${ }^{52}$ hatte sich eine besondere Weiterbildungsstätte für handwerklich erlernte Heilkunst entwickelt, vorwiegend für Anatomie und Chirurgie. Vorlesungen wurden auch in deutscher Sprache gehalten. Latein war nur für die Nomenklatur notwendig. Von 1720 an wurde der Zuzug von Schweizern immer größer, vor allem in der zweiten 
Hälfte des 18. Jahrhunderts. Es immatrikulierten sich zum Beispiel allein in den Jahren 1769 und 1770 aus der Schweiz 196 Wundärzte. Die Studierenden kamen aus den großen Orten der Ost-, West- und Zentralschweiz, aus Graubünden wie aus Glarus und Appenzell und aus kleineren Orten wie Adliswil und Wädenswil, Innertkirchen und Amsoldingen, Wiedlisbach und Lyßach, Kleindietwil und Riedtwil und vielen weiteren Ortschaften ${ }^{53}$. Diejenigen aus dem Emmental sind uns bereits bekanntgeworden. Ein ungemein fleißiges Streben nach möglichst guter Ausbildung bekundet sich hier.

\section{Die Ausbildung in Bern}

In Bern wurde in einem Gebäude des Inselspitals 1788 ein neues Sektionslokal eingerichtet; ein chemisches Laboratorium stand in der Apotheke für den Unterricht zur Verfügung, und ein botanischer Garten wurde geplant. Nur die Universität fehlte. Ein Großhöchstetter Wundarzt hatte sich in der Insel aufgehalten, bevor er 1775 nach Straßburg ging ${ }^{54}$. Den nächsten Fortschritt in Bern bildete 1797 die Gründung des Medizinisch-chirurgischen Instituts ${ }^{55}$, das trotz der politisch schwierigen Zeit sowohl von Lateinern als auch von Handwerksärzten gut besucht wurde. Schon im Winter 1797/98 nahmen zwei Handwerksärzte aus Huttwil an den Kursen teil. In den Listen von 1799 und 1800 erscheinen Emmentaler aus Langnau, Trub, Burgdorf, Heimiswil, Lützelflüh, Dürrenroth und Eriswil. Einige erhielten das Patent für Arznei- und Wundarzneikunst mit Ausnahme der Geburtshilfe und schwerer Operationen. Der prominenteste der Emmentaler war wohl 1799 Michael Schneider von Langnau, ein Urenkel Michel Schüppachs. Er ging weiter an die Universität Jena, erwarb dort am 4.Dezember 1802 das Doktordiplom und ließ sich dann in Langnau nieder ${ }^{56}$.

Bis Ende des 18. Jahrhunderts hatte sich eine wesentliche Entwicklung im Ärztewesen vollzogen. 1788 übernahm in Bern die Sanitätsbehörde, also der Staat, die Patentierung der Wundärzte und Chirurgen ${ }^{57}$. Das Collegium Insulanum wurde mit den Prüfungen beauftragt. Die Chirurgische Societät ging ein; ihre Zeit war vorbei. Sie kann für sich das Verdienst in Anspruch nehmen, daß sie während dreier Jahrhunderte mit Erfolg im praktischen ärztlichen Gebiet Richtlinien zur Ausbildung aufgestellt und durchgesetzt hat; Mißbräuche wurden von ihr gerichtlich geahndet. Sie hob damit den ärztlichen Stand auf eine ansehnliche Höhe. 


\section{Die Krankenversorgung}

In der medizinischen Versorgung des Emmentals ist noch kaum von der Unterbringung der Kranken und Invaliden die Rede gewesen. Einzelne Wundärzte nahmen Patienten zu Behandlungen in ihr Haus auf; aber die meisten wurden doch in den Familien gepflegt. Die Verlegung Unbemittelter, für die keine Familie sorgen konnte, war die Sorge der Behörden. Invaliden standen die ehemaligen Siechenhäuser in Burgdorf und Huttwil offen; in Burgdorf nahm ein Krankensaal Bettlägerige auf ${ }^{58}$. Kränkliche und Behinderte wurden oft auf den sogenannten «Umgang» geschickt, das heißt, Güterbauern abwechslungsweise zur Verköstigung oder auch zur Betreuung zugewiesen ${ }^{59}$. In Huttwil gab es im 18. Jahrhundert eine private Anstalt für Geisteskranke. Sie wurde von einem Wundarzt geführt. Sogar Patienten aus Deutschland suchten sie auf.

Für unbemittelte Schwerkranke und unhaltbare Geisteskranke wurde immer wieder um Aufnahme in die Häuser des Inselspitals gebeten. Im Burgdorfer Ratsmanual von 1734 wird das gute Ansehen der Insel erwähnt und hervorgehoben, daß sie häufig für die Burgdorfer die letzte Rettung sei. Der Langenthaler Wundarzt Jacob Mumenthaler, der in Straßburg und Berlin studiert hatte, schrieb 1755 von der Insel, daß er «in Keinem Lazareth oder Hospital solche Ordnung und Propretaet gesehen, als in diesem». Die heutige geschätzte Wirksamkeit des Inselspitals kann somit auf eine alte Tradition zurückblicken. 


\section{Anmerkungen}

(Die Manuale der Chirurgischen Societät in der Burgerbibliothek Bern sind zitiert: MCS, anschließend vor dem Schrägstrich Bandzahl, nach dem Schrägstrich Seitenzahl.)

${ }^{1}$ Die eine Rezeptsammlung befindet sich im Medizinhistorischen Institut der Universität Zürich, die andere in Privatbesitz in Langnau im Emmental. Sie sind besprochen bei MeyerSalzmann, Marta, Volksmedizin im 18. Jahrhundert. In: Berner Zeitschrift für Geschichte und Heimatkunde 1960, Nr.3/4.

${ }^{2}$ Vgl. Koelbing, Huldrych M., Renaissance der Augenheilkunde, 1540-1630, Bern 1967, S.96; Hintzsche, Erich, Albrecht von Hallers Briefe an Auguste Tissot, 1754-1777, Bern 1977, S. 365 .

3 Meyer-Salzmann, Marta, Geschichte der Medizin im Emmental, S. $47 \mathrm{f} ., 56 \mathrm{f} ., 96 \mathrm{f}$.

4 Schneider, Wolfgang, Lexikon der Arzneimittelgeschichte, Bd.2 und 3, Frankfurt 1968.

5 Meyer-Salzmann, Marta, Michel Schüppach, 1707-1781 (Berner Heimatbücher 126), Bern 1981.

${ }^{6}$ Mez, Lydia, Womit der Apotheker einst hantierte, S. 12.

7 Roth, Alfred G., Ein unbekanntes Manuskript von G.J.Kuhn. In: Burgdorfer Jahrbuch 1953. Siehe auch Anmerkung 3, S.47, 56 f.

8 Siehe Anmerkung 7.

${ }^{9}$ Balmer, Heinz, Die Herkunft des Erfinders Fritz Fischer. In: Der Schweizer Familienforscher, Nr. 32, 1965.

${ }^{10}$ Vgl. Fischer-Homberger, Esther, Geschichte der Medizin, Berlin/Heidelberg/New York 1975, S.145-153, und Michel, Theodor, Anmerkung 11, S. 15.

${ }^{11}$ Geburtshilfliche Literatur: «Der Swangern Frauwen und hebammen Rosegarten» (1513) des Chirurgen Eucharius Rößlin; das Hebammenlehrbuch des Spitalchirurgen Jacob Rueff (um 1500 geboren) erlebte zahlreiche Auflagen. Siehe auch Michel, Theodor, Bader, Scherer, Hebammen und Apotheken im alten Luzern, Diss. Basel 1931, S.16. Das im Emmental aufgefundene populärwissenschaftliche Buch lautet: «L. Christoph de Hellwig, Med. Pract. Erffurt, Neu-entdeckte Heimligkeiten Des Frauenzimmers, Bestehend in III Theilen, Wovon der Erste eine vollkommene Beschreibung von der Erzeugung der Menschen, ... bis vor- inund nach der Geburth. II. Auserlesene Curieuse Erörterte Medicinische und Physicalische Fragen sambt denen neuesten Observationibus, ... III. Von der Diaet, rechtem Gebrauch der Speise und Trank, ... Arnstadt 1725, Vierdte Auflage». - Über die Entbindungskunst siehe auch Anmerkung 33.

12 Müller, Carl, Volksmedizinisch-geburtshilfliche Aufzeichnungen aus dem Lötschental (Berner Beiträge zur Geschichte der Medizin und der Naturwissenschaften, N.F.Bd.3, Bern/Stuttgart/Wien 1969).

${ }^{13}$ Rubi, Christian, Die Irrenärzte Neukommet aus Signau. In: Archiv des Historischen Vereins des Kantons Bern, Bd. 39, H.2 (Festschrift für Richard Feller), Bern 1948, S.311-329.

${ }^{14}$ Grunau, Gustav, Hrsg. der Blätter für Bernische Geschichte, Kunst und Altertumskunde, Bd.9, Bern 1913.

${ }^{15}$ Zuber, Jakob, Armenanstalt der Einwohnergemeinde Sumiswald, 1812-1912, Sumiswald 1913, S. 80. - Das Honorar, 1 neue Dublone, entsprach nach der Umrechnungstabelle von Christian Lerch im Staatsarchiv Bern dem Wert von fast 400 Fr. für 1952/53. 
${ }^{16}$ Wieger, Friedrich, Geschichte der Medicin und ihrer Lehranstalten in Strassburg vom Jahre 1497 bis zum Jahre 1872, Strassburg 1885, S. 100-108.

17 Jenzer, Hans, Die Gründung der Hebammenschulen in der Schweiz im 18. Jahrhundert. In: Gesnerus 23 (1966) 75.

${ }_{18}$ Buess, Heinrich, Schweizer Ärzte als Forscher, Entdecker und Erfinder, Basel 1945; Balmer, Heinz, Albrecht von Haller (Berner Heimatbücher 119), Bern 1977.

19 Thurnheer, Yvonne, Die Stadtärzte und ihr Amt im alten Bern (Berner Beiträge zur Geschichte der Medizin und der Naturwissenschaften, Nr.4), Bern 1944; Wehrli, Gustav Adolf, Die Wundärzte und Bader Zürichs als zünftige Organisation (Mitteilungen der Antiquarischen Gesellschaft in Zürich, Bd.30, H.8), Zürich 1931, S.96; derselbe, Die Krankenanstalten und die öffentlich angestellten Ärzte und Wundärzte im alten Zürich (ibid., Bd.31, H.3), Zürich 1934, S.39-56.

${ }^{20}$ Siehe Anmerkung 16, S.56, und Diepgen, Paul, Geschichte der Medizin, Bd.1, Berlin 1949, S. 319 .

${ }^{21}$ Siehe Anmerkung 19, Thurnheer, S. 77.

${ }^{22}$ Anmerkung 3, S. 53-58.

${ }^{23}$ Anmerkung 5, S. 35.

24 Vgl. Anmerkung 3, S. $32 \mathrm{f}$.

${ }^{25} \mathrm{Vgl}$. Anmerkung 3, S.36-38, $59 \mathrm{f}$.

${ }^{26}$ Ihre Manuale von 1680 an sind in der Burgerbibliothek Bern vorhanden. Ebenfalls erhalten ist ihre Gesellschaftslade, eine Truhe, auf der die Trepanationsinstrumente dargestellt sind (Historisches Museum Bern).

${ }^{27}$ Schneebeli, Max, Handwerkliche Wundarzneikunst im alten Bern (Berner Beiträge zur Geschichte der Medizin und der Naturwissenschaften, Nr.9), Bern 1949, S. 49.

28 MCS 2/42; 4/130, 167.

29 Anmerkung 27, S. 38.

30 ibid., S. 44.

31 ibid., S. 70.

32 MCS 4/133.

${ }^{33}$ Über in deutscher Sprache veröffentlichte Originalwerke siehe Handbuch der Geschichte der Medizin, hrsg. von Max Neuburger und Julius Pagel, Bd.2, Jena 1903.

Aus dem Bücher-Nachlaß der Wundärzte namens Mumenthaler in Langenthal im 18. Jahrhundert seien hier herausgegriffen: Anatomische Tafeln von Julius Gasser und Daniel Bucretius, Frankfurt 1656, Anatomische Tafeln von Simon Pavelli, Frankfurt 1666, Anatomische Abhandelung von dem Bau des menschlichen Leibes von J.B. Winslow, Berlin 1733, Compendiöses doch vollkommenes anatomisches Handbüchlein von Christoph Heinrich Keil, 6.Auflage, Leipzig 1747, Osteologische Tabellen von August Schaarschmidt, Frankfurt und Leipzig 1754;

Wundarzneyisches Zeug-Hauss von Johannes Scultetus, Frankfurt 1666, Medicinische und Chirurgische Schriften von Heidenreich Overkamp, Leipzig 1705, Von den Instrumenten der Chirurgie von Jacques Croissant de Garengeot, Berlin und Potsdam 1729, Chirurgia curiosa von G.Purnau, Frankfurt und Leipzig 1739, Medicinisch, Chirurgisch, Anatomisch, Chymisch \& Botanische Abhandlungen, Nürnberg 1755 bis 1765, Anweisung zum verbesserten chirurgischen Verbande von Friedrich Henckel, 2. Auflage, Berlin und Stralsund 1767, Chirurgie von Lorenz Heister, neue Auflage, Nürnberg 1770. 
Die innere Medizin beginnt mit Grund-Regula der Medizin von Johann Helfrich Juengken, Frankfurt und Leipzig 1707. Ein weiteres Werk weist auf Sydenham: Arzney-Kunst von Michael Ettmüller und Medicinische Werke von Thomas Sydenham, Leipzig 1717. Von 1701 an folgen durch das ganze Jahrhundert medizinische Bücher vermischten Inhalts, die entweder Schatzkammer, Kunstbuch, Neu eröffneter Schatz menschlicher Gesundheit oder ähnlich heißen. Von bekannten Gelehrten werden hier aus der zweiten Hälfte des 18. Jahrhunderts noch vermerkt: Anweisung zu einer sicheren medizinischen Praxis von Friedrich Hoffmann, Ulm 1752, Phisiologie von Herman Boerhaave, übersetzt von Johann Peter Eberhard, Halle 1754, Medicus oder Consilia medica, nach Hoffmannschen und Boerhaavischen Grundsätzen, Erfurt 1762, Von der Erfahrung in der Arzneykunst von Johann Georg Zimmermann, Von der Ruhr unter dem Volke im Jahr 1765, von demselben, Zürich 1767, Grundriß der Physiologie von Albrecht von Haller, Berlin 1781, Cajus Plinius, Naturgeschichte, 9. bis 12. Band, übersetzt von Fr. Große, Frankfurt 1781.

Über die Entbindungskunst: Bruchstücke über den gewöhnlichen Hergang der Geburt, handschriftlich in acht Heften, Praktischer Unterricht in der Entbindungskunst von Johann David Busch, Marburg 1787, Magazin für Geburtshelfer, 1. Stück, Marburg 1788.

Über Botanik und Pharmazeutik: Eydgnössischer Lust-Garte von Johann von Muralt, Zürich 1715 (botanisch). Theatrum Botanicum, Das ist: Neu Vollkommenes Kräuter-Buch von Theodor Zwinger, Basel 1744, Beobachtungen, Gebrauch und Nutzen des Schierlings von Anton Störck, übersetzt aus dem Lateinischen von G.Ludewig Rumpelt, Dresden und Warschau 1762, Pharmacopoea Helvetica von Albertus de Haller, Basel 1771, Vollständiges Pflanzensystem des Ritters Carl von Linné, nach der 13.lateinischen Ausgabe, Nürnberg 1777, Deutsches Dispensatorium oder allgemeines Apothekerbuch von J.H.Pfingsten, Frankfurt und Leipzig 1783.

Nachschlagewerke sind: Thesaurus Sanitatis von J. Jb. Bräucher, Frankfurt 1710, Gelehrten-Lexikon von Burchard Markus, Leipzig 1715, Lexikon universale latino-germanicum von Theodor Spierer, Basel 1716, Der geschwinde Lateiner von Chr. Heinr. Weiß, 6. Auflage, Altenburg 1745.

Von Johann Georg Mumenthaler wurde veröffentlicht: Der Zauberarzt und Guldenes Kleinod der medizinischen Geheimnussen. Handschriftliche Aufzeichnungen haben verschiedene Mumenthaler hinterlassen: Rezeptsammlungen, darunter auch ein Heft Von den Säuren und Salzen, und Kunstbücher, alles für den eigenen Gebrauch bestimmt. Diese Dokumente und ein Teil der erwähnten Literatur sind im Heimatmuseum Langenthal noch erhalten. - Siehe auch Anmerkung 51 und die erwähnten Vorlesungen aus Straßburg.

${ }^{34}$ Anmerkung 16, S.95.

${ }^{35}$ MCS 4/21 f.

${ }^{36}$ MCS 5/412.

${ }^{37}$ MCS 5/168; hier 20 Pfund; 4 wurden ihm erlassen. 1 Bernpfund = 71/2 Batzen.

${ }^{38}$ MCS 2/138.

${ }^{39}$ MCS $3 / 500$.

${ }^{40}$ MCS 5/17.

${ }^{41}$ MCS 5/8, 167, 169, 170. Der Meisterbrief von Ulrich Kähr, ausgestellt am 12. Januar 1750, ist gedruckt in Meyer-Salzmann, vgl. Anmerkung 5, erwähnt MCS 5/87f.

${ }^{42}$ Wehrwesen, Nr. 6, Bernisches Staatsarchiv in Bern. Siehe auch Hausmann, Anmerkung 53, und MCS 6. 
43 Anmerkung 3, S. 53, 61.

${ }^{44}$ Brunner, Conrad, Die Verwundeten in den Kriegen der alten Eidgenossenschaft, Tübingen 1903.

45 Gart, Peter, Johann Ludwig Hommel (1706-1743) (Berner Beiträge zur Geschichte der Medizin und der Naturwissenschaften, Nr.14), Bern 1958.

${ }^{46}$ Anmerkung 5. Den Wortlaut von Lehrbriefen siehe dort.

${ }^{47}$ Vgl. Gotthelf, Jeremias, Anne Bäbi Jowäger.

${ }^{48}$ Koelbing, Huldrych M., Der Urin im medizinischen Denken, 7 Hefte, Basel 1967, Seite 82 ff.; Heinzmann, Joh.G., Michel Schüppach. In: Kleine Chronik für Schweizer, Bern 1795, S. $439 \mathrm{ff}$.

49 Anmerkung 16, S.72, und Notizen im Heimatmuseum Langenthal.

${ }^{50}$ Heimatmuseum Langenthal.

${ }^{51}$ Das Heimatmuseum Langenthal besitzt verschiedene weitere Belege von den Ärzten Mumenthaler, darunter eine Apotheke mit Flaschen und Töpfen, Spazierstöcke mit Medikamenten und auch Instrumente.

52 Siehe Anmerkung 16.

${ }^{53}$ Knod, Gustav C., Die Alten Matrikeln der Universität Straßburg, Bd.2 (von 1621 bis 1793), Straßburg 1897; Hausmann, S., Die Schweizer Studenten an der alten Universität Straßburg. In: Zeitschrift für Schweizerische Geschichte, Zürich 1928.

${ }^{54}$ Werder, Ernst, Aus der Geschichte des Arzneiwesens im Amt Konolfingen. In: Berner Zeitschrift für Geschichte und Heimatkunde 1947, H.2, S.90.

${ }^{55}$ Jaussi, Ruth, Das Medizinische Institut in Bern (1797-1805) (Berner Beiträge zur Geschichte der Medizin und der Naturwissenschaften, Nr.5), Bern 1944.

${ }^{56}$ Lindt, Wilhelm, Jubiläum der Medizinisch-chirurgischen Gesellschaft des Kantons Bern, Bern 1909.

${ }^{57}$ Hintzsche, Erich, Sechshundert Jahre Inselspital, 1354-1954, Bern 1954 (mit Hermann Rennefahrt), S.298.

${ }^{58}$ Anmerkung 3, S.29, 30, 38, 39, 84.

${ }^{59}$ Siehe Anmerkung 15, S.6; Vatter, Max, Das religiöse und kirchliche Leben, einschließlich des Fürsorgewesens. In: Heimatbuch des Amtes Burgdorf und der Kirchgemeinden Utzenstorf und Bätterkinden, Bd.2, Burgdorf 1938, S.409f.

${ }^{60}$ Anmerkung 3, S.63f. 


\section{Summary}

This article deals with the medical conditions in the country in the 18th century. It begins with the house medicine according to two collections of prescriptions in manuscript. The druggists could give advice for the inner medicine. Midwives were important; the Caesarian operation is mentioned. A physician who had studied at an university stole a corpse to dissect it. Usually the population was treated by barber surgeons. They had to pass an examination in the Surgical Society at Berne. Many of them went to the University of Strassburg before. Lectures in manuscript, noted by a barber surgeon, have been read.

Marta Meyer-Salzmann

Ackersteinstrasse 178

CH-8049 Zürich 